\title{
Erratum to: A Homogenized Model for Honeycomb Cellular Materials
}

\author{
Cesare Davini · Federica Ongaro
}

Published online: 23 March 2011

(C) Springer Science+Business Media B.V. 2011

\section{Erratum to: J Elast}

\section{DOI 10.1007/s10659-011-9311-z}

By an error in processing Figs. 4 and 6 the labels along the axes have not been resolved correctly. They should be read as follows

$$
T 11=\frac{1}{2}\left(\sigma_{11}^{\prime}+\sigma_{22}^{\prime}\right), \quad T 22=\frac{1}{2}\left(\sigma_{11}^{\prime}-\sigma_{22}^{\prime}\right) \quad \text { and } \quad t 12=\sigma_{12}^{\prime} .
$$

Also, in the caption of Fig. 6, read

$$
\sigma_{\alpha \beta}^{\prime}=\sigma_{\alpha \beta} /\left(\alpha^{2}\left(\frac{h}{\ell}\right)^{3} \frac{E_{s}}{\left(1-v_{s}^{2}\right)}\right) .
$$

Finally, formula (7.9) should be

$$
\eta_{1}(i) \cdot \mathbf{F}(i) \geq-\frac{\alpha^{2} \pi^{2} h^{3}}{12 \ell^{2}} \frac{E_{s}}{\left(1-v_{s}^{2}\right)} \quad i=1,2,3 .
$$

The online version of the original article can be found under doi:10.1007/s10659-011-9311-z.

C. Davini $(\bowtie) \cdot$ F. Ongaro

Dipartimento di Georisorse e Territorio, Università di Udine, Via del Cotonificio 114, 33100 Udine, Italy

e-mail: davini@uniud.it 